UCLA/94/TEP/10

hep-ph/9403313

\title{
Hadronic Spectrum in Inclusive Semileptonic B Decays
}

\author{
Antonio O. Bouzas ${ }^{1}$ and Dario Zappalà ${ }^{2}$ \\ UCLA Department of Physics \\ Los Angeles, California 90024-1547
}

\begin{abstract}
We evaluate the inclusive semileptonic B decay spectrum as a function of the final-state hadrons energy to second order in the inverse quark mass expansion and tree level in $\alpha_{s}$. We argue that there is an energy interval below the $c$ production threshold that could be used to determine $V_{u b}$.
\end{abstract}

March 1994

\footnotetext{
${ }^{1}$ Internet: bouzas@physics.ucla.edu

${ }^{2}$ Internet: zappala@physics.ucla.edu
} 
1.Introduction. The inclusive semileptonic decay of the B meson is of great importance for the determination of the Cabibbo-Kobayash-Maskawa (CKM) matrix elements $V_{c b}$ and $V_{u b}$. For this reason several attempts have been made to predict the total width and the electron energy distribution in this decay. It has recently been shown [1] that both can be computed in the framework of the QCD operator-product expansion, without resorting to models for the structure of the initial hadron. To lowest order the parton model result is recovered. Matrix elements of higher dimension operators are then expanded in powers of the inverse of the $b$ quark mass $1 / m$ by applying heavy-quark effective theory (HQET) [2].

This method has been applied [3, 国 (see also [1, 5]) to compute the electron energy spectrum to order $1 / m^{2}$. However, for values of the electron energy in the vicinity of its maximum kinematical limit the corrections to the parton model are unreliable, being dominated by large unphysical oscillations and furthermore singular at the endpoint. This fact spoils the possibility of determining $V_{u b}$, since this quantity must be extracted from the endpoint region where the $b \rightarrow c$ channel is kinematically forbidden. Some tentative solutions to this problem have been suggested such as gaussian smearing [四] or integration [3] of the spectrum in the endpoint region. Also a form factor formalism has been proposed $[6]$.

In this letter we compute the inclusive semileptonic differential decay width as a function of the total energy of the final-state hadrons employing the approach outlined above. We suggest that this quantity could be suitable for the determination of $V_{u b}$. In this case, the relevant part of the spectrum corresponds to total hadronic energies smaller than the charm mass where $b \rightarrow u$ is the only decay mode available and it is worthwhile to study the behaviour of the heavy-quark approximation scheme within this region.

2. Hadronic spectrum. In order to obtain the hadronic energy distribution we closely follow the procedure used in [4] for the electron spectrum. For that reason, we omit all technical details related to the computation. In the sequel we neglect radiative corrections of order $\alpha_{s} / \pi$.

We start from the weak hamiltonian

$$
\mathcal{H}(x)=-V_{j b} \frac{G_{F}}{\sqrt{2}} J_{l}^{\mu}(x) J_{\mu}(x)
$$

where $G_{F}$ is the Fermi constant, $V_{j b}$ is the CKM matrix element for $b \rightarrow j$ and $J_{l}^{\mu}$ and $J_{\mu}$ are the leptonic and hadronic weak currents respectively,

$$
J_{l}^{\mu}(x)=\overline{\psi_{e}}(x) \gamma^{\mu}\left(1-\gamma_{5}\right) \psi_{\nu}(x)
$$




$$
J_{\mu}(x)=\overline{\psi_{j}}(x) \gamma_{\mu}\left(1-\gamma_{5}\right) \psi_{b}(x)
$$

The squared amplitude for the semileptonic B decay can be written as ( $k$ and $k^{\prime}$ are the electron and neutrino momenta respectively, and $\left.q=k+k^{\prime}\right)$,

$$
|A|^{2}=\frac{\left|V_{j b}\right|^{2} G_{F}^{2}}{2}(2 \pi) L^{\mu \nu}\left(k, k^{\prime}\right) W_{\mu \nu}(q)
$$

The leptonic tensor $L_{\mu \nu}$ appearing in (4) is given at tree level by the expression円,

$$
\begin{aligned}
\sum_{p o l}\left\langle l, \bar{\nu}\left|J_{l}^{\nu}(x)\right| 0\right\rangle\left\langle 0\left|J_{l}^{\dagger \mu}(0)\right| l, \bar{\nu}\right\rangle=e^{i q x} L^{\mu \nu}\left(k, k^{\prime}\right) \\
=e^{i q x} 8\left(k^{\mu} k^{\prime \nu}+k^{\prime \mu} k^{\nu}-\left(k \cdot k^{\prime}\right) g^{\mu \nu}-i \epsilon^{\mu \nu \alpha \beta} k_{\alpha} k_{\beta}^{\prime}\right)
\end{aligned}
$$

The hadronic tensor, defined as,

$$
W_{\mu \nu}(q)=\frac{1}{2 \pi} \sum_{X} \int d^{4} x e^{i q x}\left\langle B\left|J_{\mu}^{\dagger}(0)\right| X\right\rangle\left\langle X\left|J_{\nu}(x)\right| B\right\rangle
$$

(where the sum runs over a complete set of hadronic states) can be related to the discontinuity of the matrix element of the time ordered product of hadronic weak currents,

$$
T_{\mu \nu}=-i \int d^{4} x e^{i q x}\left\langle B\left|T J_{\mu}^{\dagger}(0) J_{\nu}(x)\right| B\right\rangle
$$

through the relation,

$$
\operatorname{Im}\left(T_{\mu \nu} L^{\mu \nu}\right)=-\pi W_{\mu \nu} L^{\mu \nu}
$$

Finally, using (4) and (8), the B decay width is given by

$$
\Gamma_{B}=\left|V_{j b}\right|^{2} G_{F}^{2} \int \frac{d^{4} k}{(2 \pi)^{4}} \frac{d^{4} k^{\prime}}{(2 \pi)^{4}} \delta\left(k^{2}\right) \theta\left(k_{0}\right) \delta\left(k^{\prime 2}\right) \theta\left(k_{0}^{\prime}\right) \operatorname{Im}\left(-T_{\mu \nu} L^{\mu \nu}\right)
$$

with the normalization $\left\langle B\left(M_{B} v\right) \mid B\left(M_{B} v^{\prime}\right)\right\rangle=(2 \pi)^{3} v^{0} \delta^{3}\left(M_{B} v-M_{B} v^{\prime}\right)$ [4], $M_{B}$ and $v$ being the mass and velocity respectively of the $\mathrm{B}$ meson. The problem of calculating the total decay width and the leptonic and hadronic spectrum is then related to the evaluation of the tensor $T^{\mu \nu}$. We shall limit ourselves to the case $j=u$ and the $u$ quark mass will be neglected.

Due to the $T$-ordering the matrix element in (7) contains a $u$ quark propagator. In the following we shall treat separately two contributions to the quantity $T_{\mu \nu} L^{\mu \nu}$

$$
T_{\mu \nu} L^{\mu \nu}=T_{\mu \nu}^{(1)} L^{\mu \nu}+T_{\mu \nu}^{(2)} L^{\mu \nu}
$$

\footnotetext{
${ }^{1}$ We use the convention $\epsilon_{0123}=1$.
} 
where the first term contains the lowest order approximation for the $u$ propagator (i.e. the free propagator) whereas in the second one the first order correction to the propagator, consisting of a gluon insertion, is taken into account.

The first step to compute $T_{\mu \nu}^{(1)} L_{\mu \nu}$ is to factorize out the large $x$-dependence in the b field,

$$
\psi_{b}(x)=e^{-i m v x} \psi_{b}^{\prime}(x)
$$

with $m$ being the $b$ quark mass and $v$ the 4 -velocity of the $\mathrm{B}$ meson. The field on the right-hand side of (11) can be split into two components $\psi_{b}^{\prime}(x)=h(x)+\chi(x)$ defined by the properties $\phi h(x)=h(x)$ and $\not \chi \chi(x)=-\chi(x) . \chi(x)$ can be eliminated in terms of $h(x)$ through an expansion in powers of $1 / m$ [2].

At the same time, we can perform a short-distance expansion since the $x$ dependence of $\psi_{b}^{\prime}(x)$ is small in the sense that it is related to the "residual" momentum $\kappa$ defined as,

$$
\kappa=p_{b}-m v
$$

$\kappa$ is non zero for finite $m$ because the $b$ quark does not carry all of the B momentum, but it vanishes in the limit of very large quark mass.

The combination of short-distance expansion and HQET leads to a $1 / m$ expansion of the matrix element in terms of operators of increasing dimensions. The lowest order coincides with the the semileptonic decay of a free $b$ quark, since it corresponds to setting $\kappa=0$, (i.e., $p_{b}=m v$ ) and thus identifying the decaying particle with the $b$ quark. Corrections of order $1 / m$ vanish [1], whereas those of order $1 / \mathrm{m}^{2}$ are expressed in terms of the two matrix elements [3, 何]

$$
\begin{aligned}
K_{b} & =-\left\langle B\left|\bar{h}(0) \frac{(i D)^{2}}{2 m^{2}} h(0)\right| B\right\rangle \\
G_{b} & =Z_{b}\left\langle B\left|\bar{h}(0) \frac{g G^{\alpha \beta} \sigma_{\alpha \beta}}{4 m^{2}} h(0)\right| B\right\rangle
\end{aligned}
$$

$D$ being the QCD covariant derivative, $g$ the strong coupling constant, $\sigma_{\alpha \beta}=(i / 2)\left[\gamma_{\alpha}, \gamma_{\beta}\right]$, and $Z_{b} G^{\alpha \beta}$ the renormalized gluon field strength tensor. The quantity $G_{b}$ is directly related to the experimentally measured mass splitting $M\left(B^{*}\right)-M(B)$ from which one gets the small value $G_{b}=$ -0.0065 . The numerical value of $K_{b}$ is estimated to be $K_{b}=0.01$, [3, from QCD sum rules (see [3]).

The explicit expression for $T_{\mu \nu}^{(1)} L^{\mu \nu}$ to the order $1 / m^{2}$ is given by,

$$
T_{\mu \nu}^{(1)} L^{\mu \nu}=64 \int d^{4} x \int \frac{d^{4} p}{(2 \pi)^{4}} e^{-i(m v-q-p) x} \frac{1}{p^{2}+i \varepsilon}\left(R_{0}+R_{1}+R_{2}\right)
$$


where $p$ is the $u$-quark momentum and,

$$
\begin{aligned}
& R_{0}=(p \cdot k)\left(v \cdot k^{\prime}\right) \\
& R_{1}=i m\left(K_{b}+G_{b}\right) x_{\rho}\left(\frac{2}{3} k^{\prime \rho}-\frac{5}{3}\left(v \cdot k^{\prime}\right) v^{\rho}\right)(p \cdot k) \\
& R_{2}=\frac{1}{3} m^{2} K_{b}(p \cdot k)\left(v \cdot k^{\prime}\right)\left(x^{2}-(v \cdot x)^{2}\right)
\end{aligned}
$$

The discontinuity of $T_{\mu \nu}^{(1)} L^{\mu \nu}$ in (15) is given by the $u$ quark propagator

$$
\operatorname{Im}\left(\frac{1}{p^{2}+i \varepsilon}\right)=-\pi \delta\left(p^{2}\right)
$$

The integration over $x$ in (15) yields terms proportional to $\delta^{4}(m v-q-p)$ and its first two derivatives, according to the power of $x$ appearing in $R_{i}$.

We notice that each term $R_{i}$ of the expansion is symmetric in the two momenta $p$ and $k$. This symmetry can be related to a Fiertz identity for the product of currents in the amplitude that exchanges the electron and quark fields $\psi_{e}, \psi_{u}$. Thus, from (9), (15) and (16), the first piece of the decay width $\Gamma_{B}^{(1)}$ is invariant under exchange of $p$ and $k$ and the two spectra $d \Gamma_{B}^{(1)} / d E_{e}$ and $d \Gamma_{B}^{(1)} / d E_{u}$ have exactly the same form.

After performing the integrations we obtain,

$$
\begin{aligned}
\frac{1}{\Gamma} \frac{d \Gamma_{B}^{(1)}}{d w}= & {\left[6 w^{2}-4 w^{3}-\frac{20}{3} K_{b} w^{3}-\frac{20}{3} G_{b} w^{3}+4 G_{b} w^{2}\right] \theta(1-w) } \\
& +\left[\frac{2}{3} K_{b}+\frac{10}{3} G_{b}\right] \delta(1-w)+\frac{2}{3} K_{b} \delta^{\prime}(1-w)
\end{aligned}
$$

where we introduced the dimensionless variable $w=2 E_{u} / m$ and the lowest order width $\Gamma=$ $\left(\left|V_{u b}\right|^{2} G_{F}^{2} m^{5}\right) /\left(192 \pi^{3}\right)$.

Let us now consider the second term $T_{\mu \nu}^{(2)} L_{\mu \nu}$ in (10). The complete expression for $T_{\mu \nu} L^{\mu \nu}$ to order $1 / m^{2}$ includes the correction obtained by attaching a gluon line to the free $u$ propagator. The insertion of the gluon field on the propagator in the matrix element of (7) generates a term proportional to $G_{b}$ [4. Notice that this insertion is not related to the one loop self-energy correction to the propagator, whose imaginary part is proportional to the probability of real gluon emission. The new term is, then,

$$
\begin{array}{r}
T_{\mu \nu}^{(2)} L^{\mu \nu}=\frac{128}{3} \int d^{4} x \int \frac{d^{4} p}{(2 \pi)^{4}} e^{-i(m v-q-p) x}\left(\frac{1}{p^{2}+i \varepsilon}\right)^{2} m^{2} G_{b} \times \\
{\left[(v \cdot k)\left(p \cdot k^{\prime}\right)-\left(k \cdot k^{\prime}\right)(v \cdot p)\right]}
\end{array}
$$

Unlike $T_{\mu \nu}^{(1)} L_{\mu \nu}$, in the above equation the expression in square brackets is antisymmetric in $p$ and $k$. 
In fact the presence of the gluon field in the hadronic current breaks the symmetry between $\psi_{e}$ and $\psi_{u}$. In this case one can still apply a Fiertz transformation exchanging $\psi_{e}$ and the string $\psi_{u}\left(\bar{\psi}_{u} G \psi_{u}\right)$. This operation yields again the result (18), but it does not simply correspond to the exchange of $p$ and $k$ in the scalar products of (18). It also has the effect of substituting $-G_{b}$ for $G_{b}$.

In (18) the discontinuity generated by the squared propagator is,

$$
\operatorname{Im}\left(\frac{1}{p^{2}+i \varepsilon}\right)^{2}=\pi \delta^{\prime}\left(p^{2}\right)
$$

Due to the integration over $p_{0}$, the derivative acting on the delta function can be transferred to the other terms in (18). This way $\operatorname{Im}\left(T_{\mu \nu}^{(2)} L^{\mu \nu}\right)$ contains no derivatives of $\delta\left(p^{2}\right)$ but the derivative of the momentum conservation delta function has been generated. Thus, we can see that both in the first part of the calculation in (15) and in the gluon emission part (18), the potentially singular terms appear at this step of the computation as derivatives of $\delta^{4}(m v-q-p)$.

The contribution of $T_{\mu \nu}^{(2)} L^{\mu \nu}$ to the spectrum, obtained by integrating in (18) is,

$$
\frac{1}{\Gamma} \frac{d \Gamma_{B}^{(2)}}{d w}=\frac{4}{3} G_{b}\left[\left(4 w-3 w^{2}\right) \theta(1-w)-\delta(1-w)\right]
$$

As already mentioned, the gluon contribution to the leptonic spectrum has a different form. From (18) one gets $\left(y=2 E_{e} / m\right)$,

$$
\frac{1}{\Gamma} \frac{d \Gamma_{B}^{(2)}}{d y}=\frac{4}{3} G_{b}\left[-9 y^{2} \theta(1-y)+3 \delta(1-y)\right]
$$

Incidentally, we notice that both (19) and (20), although relevant in the decay energy spectra, give zero contribution to the total B decay width.

Finally, the $u$ quark decay spectrum to the order $1 / m^{2}$ is given by the sum of (17) and (19) and it is plotted in Fig. 1. Due to the presence of the delta functions we have limited the range of $w$ to $0<w<0.9$ in the figure, and to show the small $K_{b}$ dependence curves for $K_{b}=0$, and 0.05 (dotted curves) are also included. At $w=0.5$ we find that the differential width changes by about $5 \%$ as $K_{b}$ varies from 0 to 0.05 .

3.Discussion. The differential decay rate for the process $B \rightarrow e \bar{\nu} X$ is obtained, as shown above, from the discontinuity of $T_{\mu \nu} L_{\mu \nu}$ whose cut is given by the propagator of the final $u$ quark (or $c$ quark if $X$ contains charmed mesons) only. It is natural, then, to identify the total energy of the final-state hadrons $E_{X}$ with the energy carried by the $u(c)$ which is the only particle contributing 
to the imaginary part of the amplitude. This would obviously not be true had we included $O\left(\alpha_{s} / \pi\right)$ radiative corrections. In this case the propagator would be dressed by self-energy corrections, so that for instance a real gluon emission would give a new contribution to the discontinuity.

Let us now briefly comment on our results. Equations (17) and (19) show that the hadronic spectrum has the same kind of singularities at the endpoint $w=1$ that characterize the electron spectum at $y=1$. To explain this point one should notice that both hadron and electron spectra for the decay of a free $b$ quark (that is our lowest order approximation) do not vanish smoothly at the endpoint $w=1$ and $y=1$ but contain functions $\theta(1-w)$ and $\theta(1-y)$. Singularities appear in the following corrections due to expansion of those step functions [3, 6]. More explicitly, looking at (15) in momentum space, we just have the expansion of $\delta^{4}\left(p_{b}-p-q\right)$ around $\kappa=0$

$$
\delta^{4}\left(p_{b}-p-q\right)=\delta^{4}(m v-p-q)+\kappa \cdot \partial \delta^{4}(m v-p-q)+\ldots
$$

and performing the integration, due to the symmetry of the variables $p$ and $k$, the derivatives in (21) are transformed in derivatives of $\delta(1-w)$ when computing $d \Gamma_{B} / d w$ or of $\delta(1-y)$ for $d \Gamma_{B} / d y$, thus generating analogous singularities for the two cases. The same argument is valid for the singularity in the gluon insertion correction (18) since, as already discussed, it can be expressed again as a derivative on $\delta^{4}(m v-p-q)$.

Another point to be discussed concerns the region around $w=0$. As explained in [1] the discontinuity of $T^{\mu \nu} L_{\mu \nu}$ relevant to the problem considered here is given, in the $(v \cdot q)$ complex plane at fixed $q^{2}$, by a cut along the real axis in the range $\sqrt{q^{2}}<v \cdot q<\left(M_{B}^{2}+q^{2}-M_{h}^{2}\right) /\left(2 M_{B}\right)$ ( $M_{h}$ is the mass of the lightest hadron that can be produced in the $B$ decay). For large values of $q^{2}$ this cut gets closer to another cut of the amplitude, corresponding to different physical processes, defined for $v \cdot q>\left(\left(2 M_{B}+M_{h}\right)^{2}-q^{2}-M_{B}^{2}\right) /\left(2 M_{B}\right)$. More precisely when $q^{2}$ takes its maximum value $\left(M_{B}-M_{h}\right)^{2}$, the first cut is reduced to one point $v \cdot q=\left(M_{B}-M_{h}\right)$ and the other starts at $v \cdot q=M_{B}+3 M_{h}$. In our case $M_{h}$ is the pion mass and we must expect the breakdown of the expansion when $q^{2}$ is maximum. We remark that this problem is not related to the $\delta$ and $\delta^{\prime}$ in (17) and (19), those appear as soon as we allow $\kappa \neq 0$ independently of the value of $q^{2}$. In our calculation no hadronic mass $M_{h}$ appears and since all the decay products are massless, the maximum value of $q^{2}$ is $m^{2}$. In the electron spectrum we have $q^{2}=m^{2}$ at the endpoint $E_{e}=E_{e}^{\max }$. Conversely, in the case of $d \Gamma_{B} / d w$, we find $q^{2}=m^{2}$ at the opposite edge of the spectrum that is at $w=0$.

From (17) and (19), since $G_{b}$ is negative, we see that the spectrum takes negative values around 
$w=0$ in a region of the same order of magnitude as $G_{b}$. Although this is a very small effect because of the value of $G_{b}$, it is clear that our spectrum is a poor approximation to the physical one which, as a matter of fact, must be zero for any value of the energy below the first threshold.

With no information about the subsequent terms in the series, one can reasonably take $\Lambda_{Q C D}$ as an estimate of the size of the energy range where the expansion breaks down, and eventually consider the spectrum in (17), (19) as a realistic approximation for energies above, say, .8 Gev. Therefore, we suggest the possibility of using the hadronic spectrum to determine $V_{u b}$. In fact $b \rightarrow c$ contributes to the $\mathrm{B}$ decay only for energies greater than the charm mass, so that $b \rightarrow u$ can be safely considered the only allowed channel up to approximately $1.1 \mathrm{GeV}$. This means that there is an energy interval left, of about the same size as the one available at the endpoint of the electron spectrum, where the hadronic energy distribution can be of interest, being free from the problem of singularities that affects the electron spectrum at $E_{e}=E_{e}^{\max }$.

In conclusion we have evaluated the $\mathrm{B}$ decay spectrum as a function of the massless final quark energy at the $1 / m^{2}$ level and to lowest order in $\alpha_{s} / \pi$ and we have found that it shows, at the maximum energy endpoint, the same kind of singularities as the electron spectrum and, at the same time, it turns out to be unphysical also in the low energy region that is typically dominated by non perturbative effects. Still it seems that there is a small range, usable for the $V_{u b}$ determination, where the approximation holds.

Acknowledgements. We would like to thank R. Peccei for suggesting this problem. We benefited from many helpful discussions with G. Baillie, B. Hill and K. Wang.

A. O. B. is supported by an ICSC World Laboratory scholarship. D. Z. is supported by INFN.

\section{References}

[1] J. Chay, H. Georgi and B. Grinstein, Phys. Lett. B247, (1990), 399.

[2] See e.g. N. Isgur and M. Wise, "Heavy Quark Symmetry", in "B Decays", Ed. S. Stone, World Scientific, (1992); T. Mannel, W. Roberts and Z. Ryzak, Nucl. Phys. B368, (1992), 204 and references therein.

[3] I. Bigi, M. Shifman, N. Uraltsev and A. Vainshtein, Phys. Rev. Lett. 71, (1993), 496.

[4] A. Manohar and M. Wise, Phys. Rev. D49, (1994), 1310. 
[5] T. Mannel, "Operator Product Expansion for Inclusive Semileptonic Decays in Heavy Quark Effective Field Theory", Institute für Kernphysik Technische Hochschule Darmstadt Preprint IKDA 93/26, August 1993.

[6] M. Neubert, "QCD-Based Interpretation of the Lepton Spectrum in Inclusive $\bar{B} \rightarrow X_{u} \ell \bar{\nu}$ Decays", CERN Preprint CERN-TH.7087/93, November 1993. 


\section{Figure Caption.}

Differential width $d \Gamma / d w$ as a function of $w$ as given by the sum of (17) and (19). The value of the parameters is $G_{b}=-0.0065$ and $K_{b}=0.01$ (solid line), $K_{b}=0.0$ (upper dotted curve), $K_{b}=0.05$ (lower dotted curve). 
This figure "fig1-1.png" is available in "png" format from: http://arxiv.org/ps/hep-ph/9403313v1 
Hadronic Energy Spectrum

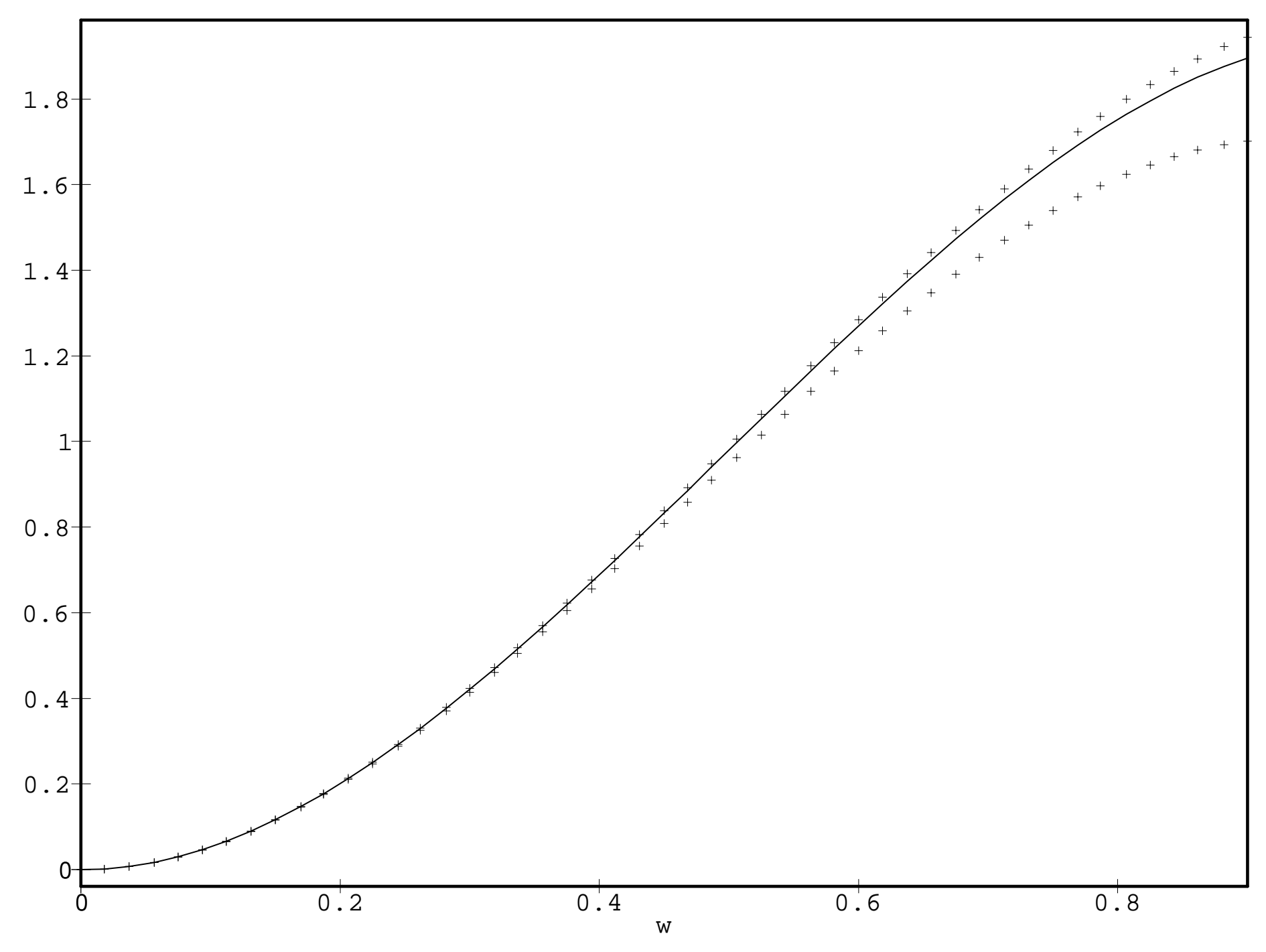

